\title{
Impact of Flow, Gradient, and Left Ventricular Function on Outcomes after Transcatheter Aortic Valve Replacement
}

\author{
Edward T. Carreras, MD¹, Tsuyoshi Kaneko, MD², Fernando Ramirez-Del Val, MD², Marc P. \\ Pelletier, MD $^{2}$, Piotr S. Sobieszczyk, MD ${ }^{1}$, Deepak L. Bhatt, MD MPH ${ }^{1}$, and Pinak B. Shah, \\ MD 1 \\ ${ }^{1}$ Cardiovascular Division, Department of Medicine, Brigham and Women's Hospital, Boston, MA \\ 2Division of Cardiac Surgery, Department of Surgery, Brigham and Women's Hospital, Boston, \\ MA
}

\section{Abstract}

Objectives-To assess the impact of low flow with and without preserved left ventricular ejection fraction (LVEF) on outcomes after transcatheter aortic valve replacement (TAVR).

\begin{abstract}
Background-Prior studies have shown that patients with low flow, AVG, and LVEF have worse outcomes after TAVR. It is unclear whether low AVG and LVEF remain prognostic after adjusting for flow, and how the outcomes of patients with low flow with and without preserved LVEF compare after TAVR. The goal of this study was to provide insight into these open questions.
\end{abstract}

Methods-Data from 340 TAVR patients at Brigham and Women's Hospital from 2011 through 2015 were analyzed. Low flow was defined as stroke volume index (SVI) $\$ 3 \mathrm{~mL} / \mathrm{m}^{2}$, low AVG as mean gradient $<40 \mathrm{mmHg}$, and reduced $\mathrm{LVEF}$ as $<50 \%$.

Results-Low flow was present in 96 (28.2\%) patients, 48 (50.0\%) of whom also had reduced LVEF. At 1 year, low flow was associated with increased mortality ( 21.9 vs $7.4 \% ; \mathrm{p}=0.0002)$ and heart failure (HF) (20.8 vs 5.3\%; $\mathrm{p}=0.0113$ ). Among patients with low flow, those with preserved LVEF had increased mortality (HR 5.17, 95\% CI 2.73-9.80; $\mathrm{p}<0.0001$ ) and HF (HR 7.69, 95\% CI 3.86-15.31; $\mathrm{p}<0.0001)$. After adjusting for clinical factors, patients with low flow had increased mortality (HR 6.51, 95\% CI 2.98-14.22; p<0.0001) and HF (HR 5.52, 95\% CI 2.34-12.98; $\mathrm{p}<0.0001$ ), while neither low AVG nor low LVEF were associated with increases in mortality or HF.

Conclusions-In patients undergoing TAVR, low flow was an independent predictor of 1-year mortality and HF, and a stronger predictor than either low AVG or LVEF. Patients with low flow and preserved EF had increased mortality and HF at 1-year, while those with low flow and reduced EF had outcomes similar to patients with normal flow.

\footnotetext{
Address for correspondence. Edward T. Carreras, MD, Brigham and Women's Hospital, 75 Francis Street, Boston, MA 02115, ecarreras@bwh.harvard.edu, 617-732-5500.

Disclosures

Dr. Carreras has no disclosures.

Dr. Kaneko is a speaker and proctor for Edwards Lifesciences.

Dr. Ramirez-Del Val has no disclosures.

Dr. Sobieszczyk has no disclosures.
} 


\section{Indexing words}

Aortic stenosis; TAVR; Stroke volume

\section{Introduction}

Transcatheter aortic valve replacement (TAVR) is an effective treatment for patients with severe aortic stenosis (AS) at intermediate, high, or prohibitive risk of major complications with surgical aortic valve replacement (SAVR).(1-3) As the use of TAVR expands, it is helpful for clinicians to understand which patients are likely to derive the most benefit and which are likely to be at highest risk for adverse outcomes. Traditional severe AS is defined by major societal guidelines as an aortic valve area (AVA) $<1.0 \mathrm{~cm}^{2}$, a peak transaortic velocity $>4.0 \mathrm{~m} / \mathrm{s}$, and a mean transaortic gradient $>40 \mathrm{mmHg}$.(4,5) However, patients with severe AS frequently have discordant echocardiographic findings, some of which have been shown to carry prognostic significance.

Reduced left ventricular ejection fraction (LVEF) has previously been associated with worse outcomes after SAVR. $(6,7)$ However, recent studies have shown that patients with reduced LVEF have similar outcomes to those with preserved LVEF after TAVR. $(6,8,9)$ Low aortic valve gradient (AVG) has been associated with poor outcomes after both TAVR and SAVR when compared to patients with normal AVG. $(7,9,10)$ When low LVEF and AVG have been studied together in a modern TAVR population, low AVG was independently associated with higher rates of mortality and heart failure (HF) readmission after TAVR, whereas low LVEF was not an independent predictor of poor outcomes.(9)

Reduced transaortic flow, as measured by the stroke volume index, is another hemodynamic parameter associated with poor outcomes in patients with severe AS, and prior studies have suggested that patients with preserved LVEF and both low flow and low AVG (Stage D3 AS per the ACC/AHA classification system) experience the worst outcomes when compared to other flow and gradient profiles.(7,11-15) This 'paradoxical' low flow, low AVG state is associated with a small ventricular cavity size, poor diastolic filling, and concentric hypertrophy, and may be indicative of alternative processes that are not entirely modified by AVR. $(7,12)$ With respect to TAVR, a few studies have similarly found that low flow is associated with increased mortality and HF readmission after TAVR, and may be a stronger independent predictor than either low AVG or LVEF, which may be less prognostic when adjusting for flow. $(11,14)$

As TAVR use has expanded to a greater range of patients, there are limited data on how low flow compares with AVG and LVEF in predicting poor outcomes in a modern TAVR population, and it is unclear how the outcomes of patients with low flow and preserved LVEF compare with those of patients with low flow and reduced LVEF. To examine these questions, we collected hemodynamic parameters and analyzed outcomes from a TAVR population at Brigham and Women's Hospital. 


\section{Materials and Methods}

\section{Study Design}

There were 375 consecutive patients who underwent TAVR at Brigham and Women's Hospital from November 2011 through July 2015. Of these, patients with incomplete baseline echocardiographic data or loss to follow up were excluded. Patient characteristics, preoperative data, laboratory test results and in hospital outcomes were recorded at the time of presentation. Data were extracted from the hospital's electronic medical records and defined according to the STS/ACC Transcatheter Valve Therapy (TVT) Registry Coder's Data Dictionary v2.0, unless otherwise noted. AVG and LVEF were obtained from transthoracic echocardiogram reports. Stroke volume index was obtained from the reports when specified. When stroke volume index was not specified in the reports, it was calculated using the reported left ventricular outflow tract (LVOT) velocity time integral and LVOT diameter. Low flow was defined as a stroke volume index less than or equal to $35 \mathrm{~mL} / \mathrm{m}^{2}$. Low AVG was defined as a mean AVG less than $40 \mathrm{mmHg}$. Low LVEF was defined as LVEF less than $50 \%$. Mortality at 30 days was defined as any in-hospital death during the index admission, or within 30 days of TAVR, if discharged. Follow-up time was calculated in months from the TAVR procedure date to the date of death or December 2016, and censored at last known clinical contact. The primary endpoint was mortality at 1 year. Secondary endpoints included readmission for HF at 1 year, and mortality and HF readmission at 30 days. In-hospital outcomes included mortality, stroke, mean change in creatinine, need for blood transfusion, and lengths of intensive care and hospital admission. The Kansas City Cardiomyopathy Questionnaire (KCCQ) scores at baseline and 30-days were also analyzed. The Brigham and Women's Hospital Institutional Review Board approved this study.

\section{Statistical Analysis}

Normally distributed continuous variables are expressed as means and standard deviation and were compared using Student's t-tests with Levene's test for homogeneity of variance. Non-normally distributed variables are expressed as median and inter-quartile range (IQR) and were compared using Mann-Whitney $\mathrm{U}$ tests. Categorical variables are presented as frequencies and percentages and compared using $\chi 2$ or Fisher's exact tests. Longitudinal time-to-event outcomes were estimated by Kaplan-Meier analyses. Adjusted time-to-event analyses were performed using forward stepwise COX proportional hazards models with a 0.1 significance level to enter and 0.05 significance level to stay. Covariates included in the models included flow, AVG, LVEF, age, sex, diabetes, hypertension, atrial fibrillation, peripheral artery disease, prior stroke, current dialysis, mean creatinine, prior coronary stent, prior coronary artery bypass graft surgery, prior pacemaker, prior valve procedure, chronic lung disease, New York Heart Association functional class, current coronary disease, STS score, access site, transcatheter valve type, and valve-in-valve TAVR. All analyses were conducted using SAS Software version 9.4 (SAS Institute, Cary, NC) and p $₫ . .05$ was the criterion for statistical significance. 


\section{Results}

A total of 340 patients who underwent TAVR for severe AS at Brigham and Women's Hospital from November 2011 through July 2015 were analyzed (Figure 1). Baseline, echocardiographic, and procedural characteristics stratified according to flow state are shown in Table 1. Most baseline and procedural characteristics were similar between groups, with significant differences in rate of prior pacemaker placement and valve type. With respect to echocardiographic characteristics, patients with low flow had lower mean LVEF (45.9 vs. $55.4 \%$; p $<0.001)$ and lower mean AVG (35.1 vs. $46.0 \mathrm{mmHg}$; $\mathrm{p}<0.001)$. With reduced LVEF defined as $<50 \%$ and low mean AVG as $<40 \mathrm{mmHg}$, patients with low flow had a significantly higher rate of reduced LVEF (50 vs. $16.4 \%$; $\mathrm{p}<0.001$ ) and low mean AVG (71.9 vs. $26.2 \%$; $<<0.0001)$.

With respect to in-hospital outcomes, patients with low flow had significantly higher rates of mortality ( $12.5 \%$ vs. $2.1 \%$, p $<0.001)$ and need for blood transfusion $(32.3 \%$ vs. $18.4 \%$, $\mathrm{p}=0.0029$ ), in addition to longer durations of hospital admission ( 7.1 vs. 4.9 days; $\mathrm{p}=0.0041$ ) and time spent in an intensive care unit (63.5 vs. 35.7 hours; $\mathrm{p}=0.024$ ) (Table 2). At 30 days, patients with low flow had significantly higher rates of mortality (15.6 vs. $2.5 \%$; $\mathrm{p}<0.001$ ) and readmission for HF (8.6 vs. $2.1 \%$; $\mathrm{p}=0.011$ ) (Table 3).

In the unadjusted analysis, patients with low flow had significantly higher rates of mortality (21.9 vs. $7.4 \%$; HR 3.42, 95\% CI 1.82-6.43; $\mathrm{p}<0.001)$ and readmission for HF (20.8 vs. $5.3 \%$; HR 5.09, 95\% CI 2.53-10.26; p<0.001) at 1 year (Figure 2). Patients with low AVG had significantly higher rates readmission for HF but no increase in mortality, and patients with low LVEF had no differences in mortality or readmission for HF (Figure 3). Among patients with low flow, those with reduced LVEF had outcomes similar to patients with normal flow (HR 0.94, 95\% CI 0.37-2.40; $\mathrm{p}=0.89$ for mortality), while those with preserved LVEF experienced an increased risk of mortality (HR 5.17, 95\% CI 2.73-9.80; $<<0.001$ ) and HF readmission (HR 7.69, 95\% CI 3.86-15.31; p<0.001) compared with patients with normal flow (Figure 4). Conversely, there were no significant differences in mortality or HF readmission when comparing patients with low flow and low AVG to those with low flow and high AVG.

In the adjusted analysis, patients with low flow had significantly higher risks of mortality (HR 6.51, 95\% CI 2.98-14.22; $\mathrm{p}<0.001$ ) and readmission for HF (HR 5.52, 95\% CI 2.3412.98; $\mathrm{p}<0.001$ ) at 1 year (Figure 5). Low AVG was not significantly associated with either mortality or HF readmission at 1 year in the adjusted analysis, while low LVEF was associated with decreased mortality (HR $0.35 ; 95 \%$ CI $0.15-0.79 ; \mathrm{p}=0.012$ ).

With respect to health status outcomes, KCCQ scores were available at both baseline and at 30-day follow up in 23 of the low flow patients and 135 of the preserved flow patients. The mean change in KCCQ score was 38.5 (SD 34.0) for patients with low flow and 40.1 (SD 27.7) for patients with preserved flow ( $\mathrm{p}=0.81$ ). With respect to $\mathrm{AVG}$, the mean change in KCCQ score was 36.3 (SD 26.3) for patients with low gradient and 41.6 for patients with preserved gradient $(\mathrm{p}=0.27)$. With respect to LVEF, the mean change in KCCQ score was 48.8 for patients with low LVEF and 37.2 for patients with preserved LVEF $(\mathrm{p}=0.032)$. 
Among those with reduced LVEF, data from follow up echocardiograms at 30 days after TAVR were available in $42(47.7 \%)$ patients, of whom 27 had an at least $10 \%$ improvement in LVEF (mean improvement in LVEF 15.2\%, SD 7.4\%). Among the patients with baseline reduced LVEF and available 30-day LVEF data, improvement in LVEF of at least $10 \%$ was associated with numerically lower but not significant differences in mortality (11.1 vs $13.3 \%, \mathrm{p}=0.36)$ and $\mathrm{HF}$ readmission ( 8.3 vs $21.4 \%$; $\mathrm{p}=0.20)$.

\section{Discussion}

In this study, low transaortic flow was associated with increased mortality and HF readmission at 1 year after TAVR. Furthermore, among patients with low flow, the rates of morality and HF were highest among those with preserved LVEF. After adjustment for clinical factors, low flow, but not low AVG or LVEF, was associated with increased 1-year mortality and HF readmission, while low LVEF was associated with a reduction in 1-year mortality. Finally, reduced LVEF, but not low flow or AVG, predicted improved health status outcomes at 30 days.

The findings that low flow predicted increased mortality and HF readmission after TAVR, and that low flow was a stronger predictor than either low AVG or LVEF, are consistent with and build upon the results of prior studies. $(7,11,13,14)$ Most studies that have shown reduced LVEF and AVG to be associated with an increase in mortality and HF after TAVR have not fully adjusted for stroke volume. $(9,16)$ However, studies that have adjusted for stroke volume have similarly found that reduced LVEF and AVG are not independently associated with mortality and HF after TAVR.(11,14) The finding that patients with low flow had a greater need for blood transfusion was unexpected. It is possible that this group had a higher rate of vascular complications, which could account in part for their worse in-hospital outcomes, though this has not been previously seen.(11) Alternatively, it may be that the hemodynamic profile and propensity for heart failure in low flow patients after TAVR predisposed them to longer hospital stays and that the need for blood transfusions was secondary to longer and more complicated hospitalizations.

Conversely, the finding that patients with low flow and preserved LVEF do worse than those with reduced LVEF has not previously been demonstrated. $(10,11,13,17)$ The pathology and hemodynamics associated with low flow and preserved LVEF, including myocardial fibrosis, increased left ventricular afterload, restrictive physiology and reduced left ventricular diastolic filling may be less easily modified by AVR.(11,18,19) Still, prior studies have shown either no association between low flow and LV function or improved outcomes among those with preserved LVEF. $(10,11,13,17)$ There are several possible explanations for the findings in this study. TAVR is associated with a higher incidence of paravalvular regurgitation compared with SAVR. Moderate to severe paravalvular regurgitation has been shown to be associated with worse outcomes.(20) Paravalvular regurgitation may be particularly poorly tolerated in patients with low flow and preserved LVEF, and a higher incidence among those patients in this study may be partially responsible for the results.

Another possible explanation for the poor outcomes among patients with low flow and preserved LVEF is undiagnosed amyloidosis. Prior studies have shown that a significant 
proportion of patients with heart failure with preserved LVEF have undiagnosed senile amyloidosis. $(21,22)$ In addition, senile amyloidosis and calcific aortic stenosis share a common demographic, and studies have described their coexistence in patients.(23) Amyloidosis is associated with a restrictive cardiomyopathy, characterized by biventricular wall thickening, loss of ventricular elasticity, and impaired ventricular relaxation.(24) While there are no studies evaluating the outcomes of TAVR in patients with amyloidosis and AS, this hemodynamic profile may be unlikely to benefit from TAVR, and may be particularly poorly suited to tolerate paravalvular regurgitation.

Another possible explanation for the difference in outcomes among low flow patients with preserved and reduced LVEF may be that the patients with reduced LVEF in fact did better than expected. Low flow may be a marker of more severe and less reversible contractile dysfunction, explaining why in prior studies, these patients have done similarly to those with preserved LVEF, and worse than those with normal flow. In the current study, patients with reduced LVEF did better overall compared with prior TAVR populations, with an overall improvement in adjusted survival. If the benefit of AVR in this population is linked to contractile reserve, it is possible that patients in this study with reduced LVEF may have had a higher than average proportion of contractile reserve, leading them to benefit more from TAVR and exaggerating the difference in outcomes seen among reduced and preserved LVEF patients with low flow. While we did not see an association between poor outcomes and an increase in LVEF at 30 days, follow up echocardiograms were only available in fewer than half of the patients with baseline reduced LVEF, some of whom already experienced an event prior to 30 days.

While the survival benefit for all patients with reduced LVEF was unexpected, the findings that these patients did not suffer increased mortality or HF readmission, and the association with improved health status outcomes, is consistent with some prior studies. $(9,11)$ These data further support the safety and efficacy of TAVR in patients with reduced LVEF. Likewise, while low flow may be a predictor of poorer outcomes after TAVR, there is ample data to suggest that these patients do indeed still benefit from AVR and that AVR is superior to medical therapy. $(10,11,25)$ As such, while flow may be a useful prognostic tool, it should not prohibit the offering of TAVR.

\section{Study Limitations}

There are several notable limitations to our study. First, this is a retrospective, observational study of patients treated at a single institution, limiting the generalizability of the results and exposing the results to potential bias. Second, several clinical parameters were not widely available, such as frailty, and as such were not included in the model and may be confounding the results. Third, the echocardiograms were all interpreted within a single echocardiography laboratory, and as such, the determination of left ventricular ejection fraction and calculation of the stroke volume index may be influenced by the customs of a single lab, such as the routine measurement of the LVOT diameter. Fourth, preoperative testing for contractile reserve, such as dobutamine stress echocardiograms, which is known to impact outcomes after AVR, were not available for most patients.(26) Fifth, the majority of patients in this cohort were treated with early generation TAVR devices that were 
associated with moderate to severe paravalvular regurgitation in upwards of $10 \%$ of patients. Patients with low flow, low AVG, and preserved LVEF may be more susceptible to the adverse outcomes associated with paravalvular regurgitation given presumed altered myocardial physiology. Whether these worse outcomes would be seen in a cohort of patients treated with later generation TAVR devices designed to minimize paravalvular regurgitation is not known. Finally, many of our patients were followed at other institutions and our knowledge of their follow up course came from phone calls; as such, it is possible that some follow up events were not captured.

\section{Future Research}

There are several potential directions for this work moving forward. One area that warrants increased attention is the routine method of determining the stroke volume index, which is the product of the LVOT area and flow, divided by the body surface area. The LVOT flow is measured by the velocity-time integral Doppler profile, while the area is determined by measuring the LVOT diameter, usually in the two-dimensional parasternal long axis, and then calculating the area assuming the LVOT is a perfect circle. There are multiple potential measurement errors and incorrect assumptions involved in this process, and in the future, it would be interesting to directly measure the LVOT area by CT scan and see how well this correlated with echocardiographic stroke volume index determination, and how, if at all, the association between flow and outcomes is affected.

Another potential area of future interest is whether the association between flow and outcomes remains in lower risk patients. Interestingly, preliminary data recently presented from a substudy of the PARTNER IIA trial reported no difference in mortality among patients with low flow and low AVG with either preserved or reduced LVEF.(27) This was the first study to assess these parameters and outcomes in a large cohort of intermediate-risk TAVR patients. One possible hypothesis is that the association with low flow and poor outcomes is stronger in higher risk patients due to the presence of confounding conditions, such as frailty or undiagnosed myocardial diseases (i.e., amyloidosis), which may be less prevalent in the lower risk cohort. Another possible explanation is the increased use of newer generation valves with a lower incidence of paravalvular regurgitation. As such, as the use of TAVR expands into lower risk patients, it will be interesting to see if the association between low flow and poor outcomes remains.

\section{Conclusion}

Low transaortic flow is associated with increased mortality and HF after TAVR and is a stronger independent predictor for outcomes than either low AVG or reduced LVEF. In this study, the poor outcomes of patients with low flow were driven by those with preserved LVEF, while patients with low flow and reduced LVEF had similar outcomes to patients with normal flow. Stroke volume index should be part of the routine preoperative evaluation of patients with AS.

\section{Acknowledgments}

Dr. Pelletier has worked as a consultant/proctor for Medtronic and St. Jude Medical. 
Dr. Bhatt discloses the following relationships: Advisory Board: Cardax, Elsevier Practice Update Cardiology, Medscape Cardiology, Regado Biosciences; Board of Directors: Boston VA Research Institute, Society of Cardiovascular Patient Care; Chair: American Heart Association Quality Oversight Committee; Data Monitoring Committees: Cleveland Clinic, Duke Clinical Research Institute, Harvard Clinical Research Institute, Mayo Clinic, Population Health Research Institute; Honoraria: American College of Cardiology (Senior Associate Editor, Clinical Trials and News, ACC.org), Belvoir Publications (Editor in Chief, Harvard Heart Letter), Duke Clinical Research Institute (clinical trial steering committees), Harvard Clinical Research Institute (clinical trial steering committee), HMP Communications (Editor in Chief, Journal of Invasive Cardiology), Journal of the American College of Cardiology (Guest Editor; Associate Editor), Population Health Research Institute (clinical trial steering committee), Slack Publications (Chief Medical Editor, Cardiology Today's Intervention), Society of Cardiovascular Patient Care (Secretary/Treasurer), WebMD (CME steering committees); Other: Clinical Cardiology (Deputy Editor), NCDR-ACTION Registry Steering Committee (Chair), VA CART Research and Publications Committee (Chair); Research Funding: Amarin, Amgen, AstraZeneca, Bristol-Myers Squibb, Chiesi, Eisai, Ethicon, Forest Laboratories, Ironwood, Ischemix, Lilly, Medtronic, Pfizer, Roche, Sanofi Aventis, The Medicines Company; Royalties: Elsevier (Editor, Cardiovascular Intervention: A Companion to Braunwald's Heart Disease); Site CoInvestigator: Biotronik, Boston Scientific, St. Jude Medical (now Abbott); Trustee: American College of Cardiology; Unfunded Research: FlowCo, Merck, PLx Pharma, Takeda.

Dr. Shah has received honoraria from Edwards Lifesciences and St. Jude Medical.

Funding Sources:

This study was funded in part by the Levinger Family Gift for Heart Valve Repair at Brigham and Women's Hospital and in part by grant T32 HL007604 from the National Institutes of Health.

\section{Abbreviations}

$\begin{array}{ll}\text { AS } & \text { Aortic stenosis } \\ \text { TAVR } & \text { Transcatheter aortic valve replacement } \\ \text { SAVR } & \text { surgical aortic valve replacement } \\ \text { AVG } & \text { aortic valve gradient } \\ \text { LVEF } & \text { left ventricular ejection fraction } \\ \text { HF } & \text { heart failure } \\ \text { LVOT } & \text { left ventricular outflow tract }\end{array}$

\section{References}

1. Leon MB, Smith CR, Mack M, et al. Transcatheter aortic-valve implantation for aortic stenosis in patients who cannot undergo surgery. N Engl J Med. 2010; 363:1597-607. [PubMed: 20961243]

2. Smith CR, Leon MB, Mack MJ, et al. Transcatheter versus surgical aortic-valve replacement in high-risk patients. N Engl J Med. 2011; 364:2187-98. [PubMed: 21639811]

3. Leon MB, Smith CR, Mack MJ, et al. Transcatheter or Surgical Aortic-Valve Replacement in Intermediate-Risk Patients. N Engl J Med. 2016; 374:1609-20. [PubMed: 27040324]

4. Erbel R, Aboyans V, Boileau C, et al. 2014 ESC Guidelines on the diagnosis and treatment of aortic diseases: Document covering acute and chronic aortic diseases of the thoracic and abdominal aorta of the adult. The Task Force for the Diagnosis and Treatment of Aortic Diseases of the European Society of Cardiology (ESC). Eur Heart J. 2014; 35:2873-926. [PubMed: 25173340]

5. Nishimura RA, Otto CM, Bonow RO, et al. 2014 AHA/ACC guideline for the management of patients with valvular heart disease: a report of the American College of Cardiology/American Heart Association Task Force on Practice Guidelines. J Am Coll Cardiol. 2014; 63:e57-185. [PubMed: 24603191]

6. Halkos ME, Chen EP, Sarin EL, et al. Aortic valve replacement for aortic stenosis in patients with left ventricular dysfunction. Ann Thorac Surg. 2009; 88:746-51. [PubMed: 19699891] 
7. Pibarot P, Dumesnil JG. Low-flow, low-gradient aortic stenosis with normal and depressed left ventricular ejection fraction. J Am Coll Cardiol. 2012; 60:1845-53. [PubMed: 23062546]

8. van der Boon RM, Nuis RJ, Van Mieghem NM, et al. Clinical outcome following Transcatheter Aortic Valve Implantation in patients with impaired left ventricular systolic function. Catheter Cardiovasc Interv. 2012; 79:702-10. [PubMed: 22121094]

9. Baron SJ, Arnold SV, Herrmann HC, et al. Impact of Ejection Fraction and Aortic Valve Gradient on Outcomes of Transcatheter Aortic Valve Replacement. J Am Coll Cardiol. 2016; 67:2349-58. [PubMed: 27199058]

10. O'Sullivan CJ, Stortecky S, Heg D, et al. Clinical outcomes of patients with low-flow, low-gradient, severe aortic stenosis and either preserved or reduced ejection fraction undergoing transcatheter aortic valve implantation. Eur Heart J. 2013; 34:3437-50. [PubMed: 24096324]

11. Herrmann HC, Pibarot P, Hueter I, et al. Predictors of mortality and outcomes of therapy in lowflow severe aortic stenosis: a Placement of Aortic Transcatheter Valves (PARTNER) trial analysis. Circulation. 2013; 127:2316-26. [PubMed: 23661722]

12. Pibarot P, Dumesnil JG. Paradoxical low-flow, low-gradient aortic stenosis: new evidence, more questions. Circulation. 2013; 128:1729-32. [PubMed: 24048202]

13. Eleid MF, Sorajja P, Michelena HI, Malouf JF, Scott CG, Pellikka PA. Flow-gradient patterns in severe aortic stenosis with preserved ejection fraction: clinical characteristics and predictors of survival. Circulation. 2013; 128:1781-9. [PubMed: 24048203]

14. Le Ven F, Freeman M, Webb J, et al. Impact of low flow on the outcome of high-risk patients undergoing transcatheter aortic valve replacement. J Am Coll Cardiol. 2013; 62:782-8. [PubMed: 23770162]

15. Nishimura RA, Otto CM, Bonow RO, et al. 2014 AHA/ACC Guideline for the Management of Patients With Valvular Heart Disease: a report of the American College of Cardiology/American Heart Association Task Force on Practice Guidelines. Circulation. 2014; 129:e521-643. [PubMed: 24589853]

16. Eleid MF, Goel K, Murad MH, et al. Meta-Analysis of the Prognostic Impact of Stroke Volume, Gradient, and Ejection Fraction After Transcatheter Aortic Valve Implantation. Am J Cardiol. 2015; 116:989-94. [PubMed: 26195275]

17. Lauten A, Figulla HR, Mollmann H, et al. TAVI for low-flow, low-gradient severe aortic stenosis with preserved or reduced ejection fraction: a subgroup analysis from the German Aortic Valve Registry (GARY). EuroIntervention. 2014; 10:850-9. [PubMed: 25415152]

18. Cramariuc D, Cioffi G, Rieck AE, et al. Low-flow aortic stenosis in asymptomatic patients: valvular-arterial impedance and systolic function from the SEAS Substudy. JACC Cardiovasc Imaging. 2009; 2:390-9. [PubMed: 19580719]

19. Herrmann S, Stork S, Niemann M, et al. Low-gradient aortic valve stenosis myocardial fibrosis and its influence on function and outcome. J Am Coll Cardiol. 2011; 58:402-12. [PubMed: 21757118]

20. Kodali SK, Williams MR, Smith CR, et al. Two-year outcomes after transcatheter or surgical aortic-valve replacement. N Engl J Med. 2012; 366:1686-95. [PubMed: 22443479]

21. Gonzalez-Lopez E, Gallego-Delgado M, Guzzo-Merello G, et al. Wild-type transthyretin amyloidosis as a cause of heart failure with preserved ejection fraction. Eur Heart J. 2015; 36:2585-94. [PubMed: 26224076]

22. Mohammed SF, Mirzoyev SA, Edwards WD, et al. Left ventricular amyloid deposition in patients with heart failure and preserved ejection fraction. JACC Heart Fail. 2014; 2:113-22. [PubMed: 24720917]

23. Longhi S, Lorenzini M, Gagliardi C, et al. Coexistence of Degenerative Aortic Stenosis and WildType Transthyretin-Related Cardiac Amyloidosis. JACC Cardiovasc Imaging. 2016; 9:325-7. [PubMed: 26189123]

24. Guan J, Mishra S, Falk RH, Liao R. Current perspectives on cardiac amyloidosis. Am J Physiol Heart Circ Physiol. 2012; 302:H544-52. [PubMed: 22058156]

25. Mohty D, Magne J, Deltreuil M, et al. Outcome and impact of surgery in paradoxical low-flow, low-gradient severe aortic stenosis and preserved left ventricular ejection fraction: a cardiac catheterization study. Circulation. 2013; 128:S235-42. [PubMed: 24030412] 
26. Barbash IM, Minha S, Ben-Dor I, et al. Relation of preprocedural assessment of myocardial contractility reserve on outcomes of aortic stenosis patients with impaired left ventricular function undergoing transcatheter aortic valve implantation. Am J Cardiol. 2014; 113:1536-42. [PubMed: 24641965]

27. Clavel, M., Pibarot, P., Gertz, Z., et al. American College of Cardiology Scientific Sessions 2017. Washinton, D.C: 2017. Outcomes of the Difference Flow/Gradient Patterns of Aortic Stenosis After Aortic Valve Replacement: Insights from PARTNER IIA. 


\section{TAVR patients}

$$
\mathrm{N}=375
$$

\section{Missing echo data}

$$
\mathrm{N}=14
$$

\section{Lost to follow up}

$\mathrm{N}=21$

Figure 1. Study flow chart

Flow chart illustrating the derivation of the study population. TAVR, transcatheter aortic valve replacement. 
A

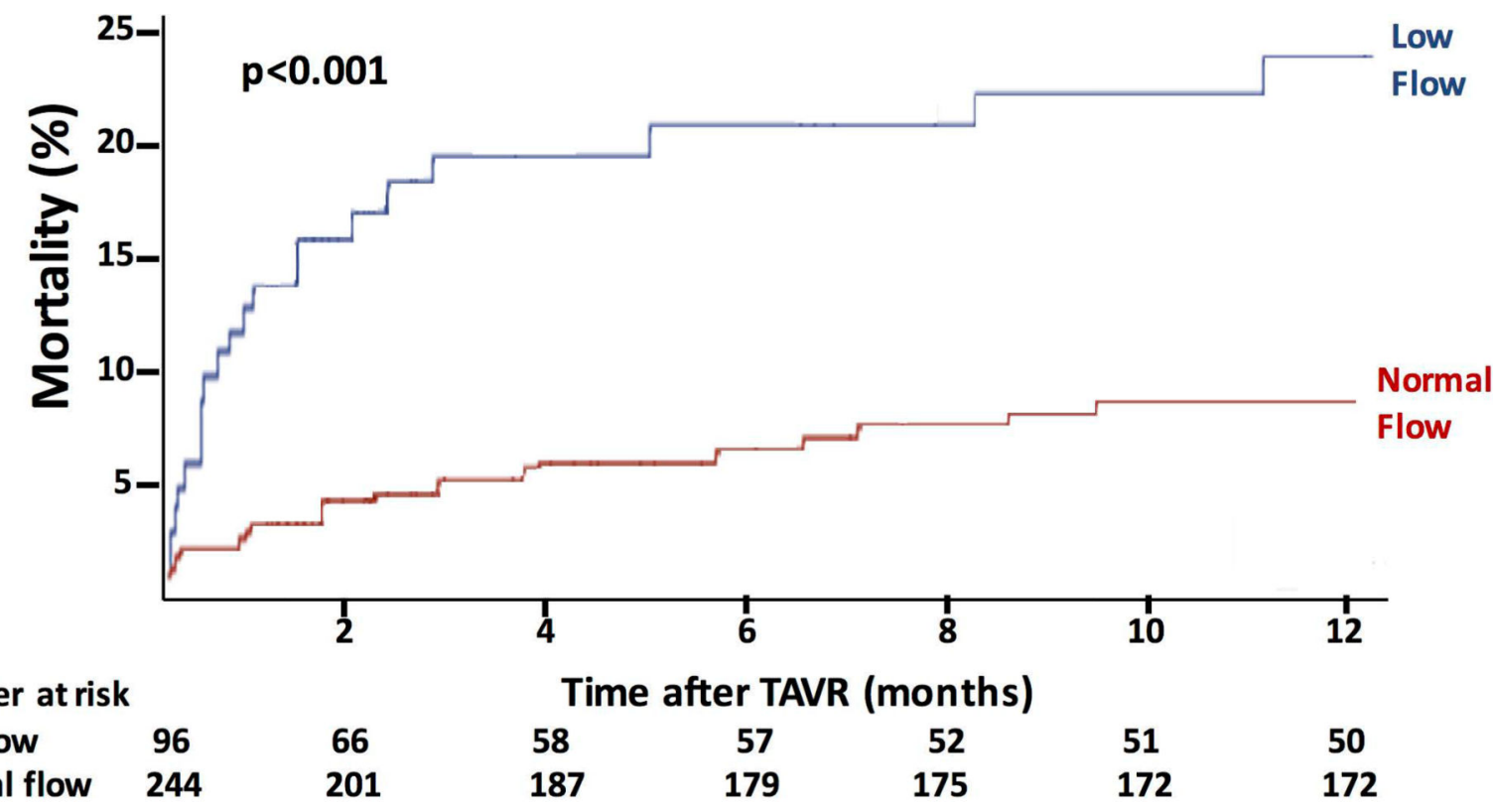

Number at risk

Low flow $\quad 96$

Normal flow 244

201

187

179

172

172

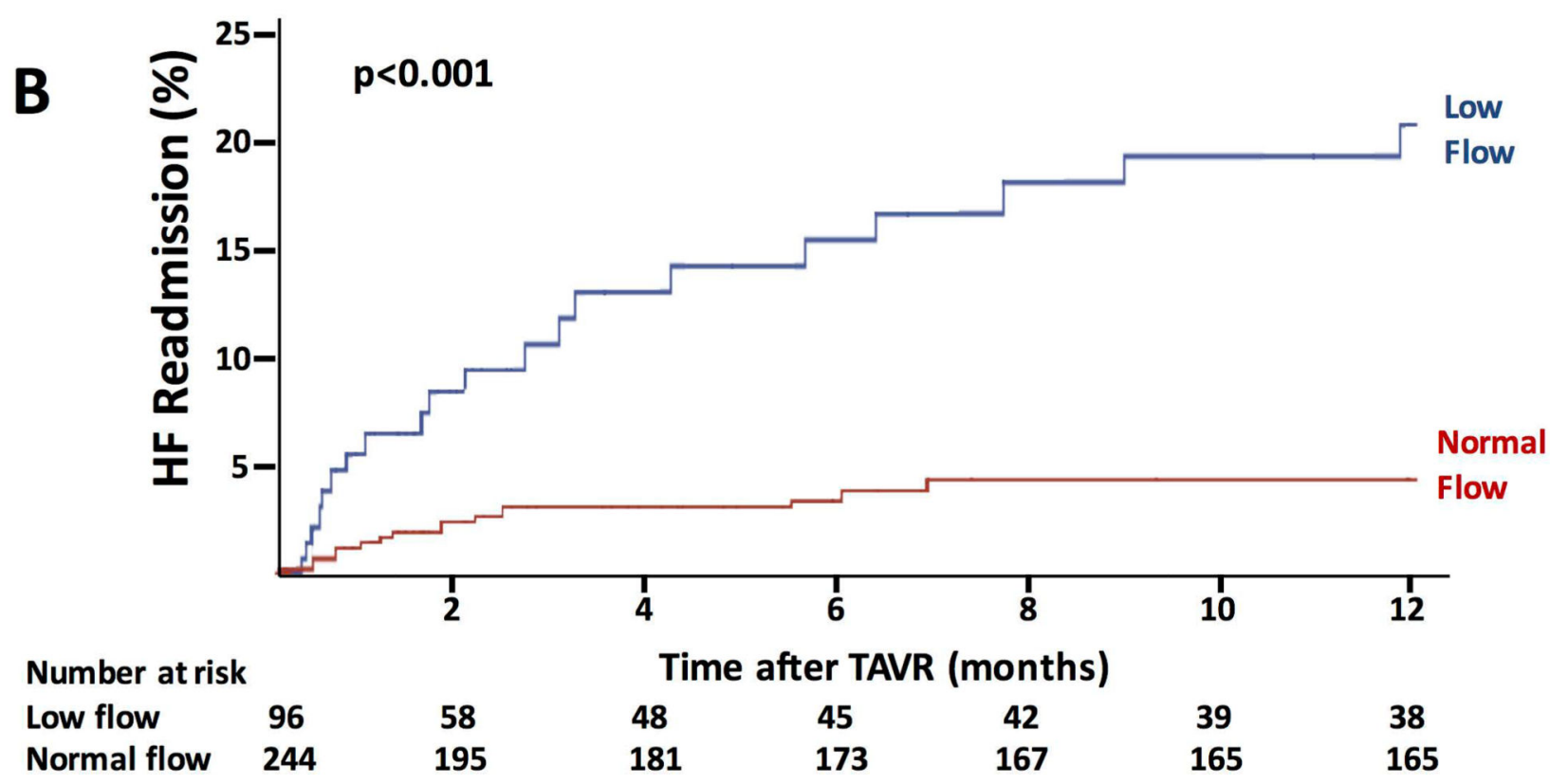

Figure 2. Cumulative incidence of mortality and heart failure readmission stratified by flow state Kaplan-Meier survival curves for mortality (panel A) and heart failure readmission (panel B) stratified by flow state. HF, heart failure; TAVR, transcatheter aortic valve replacement. 


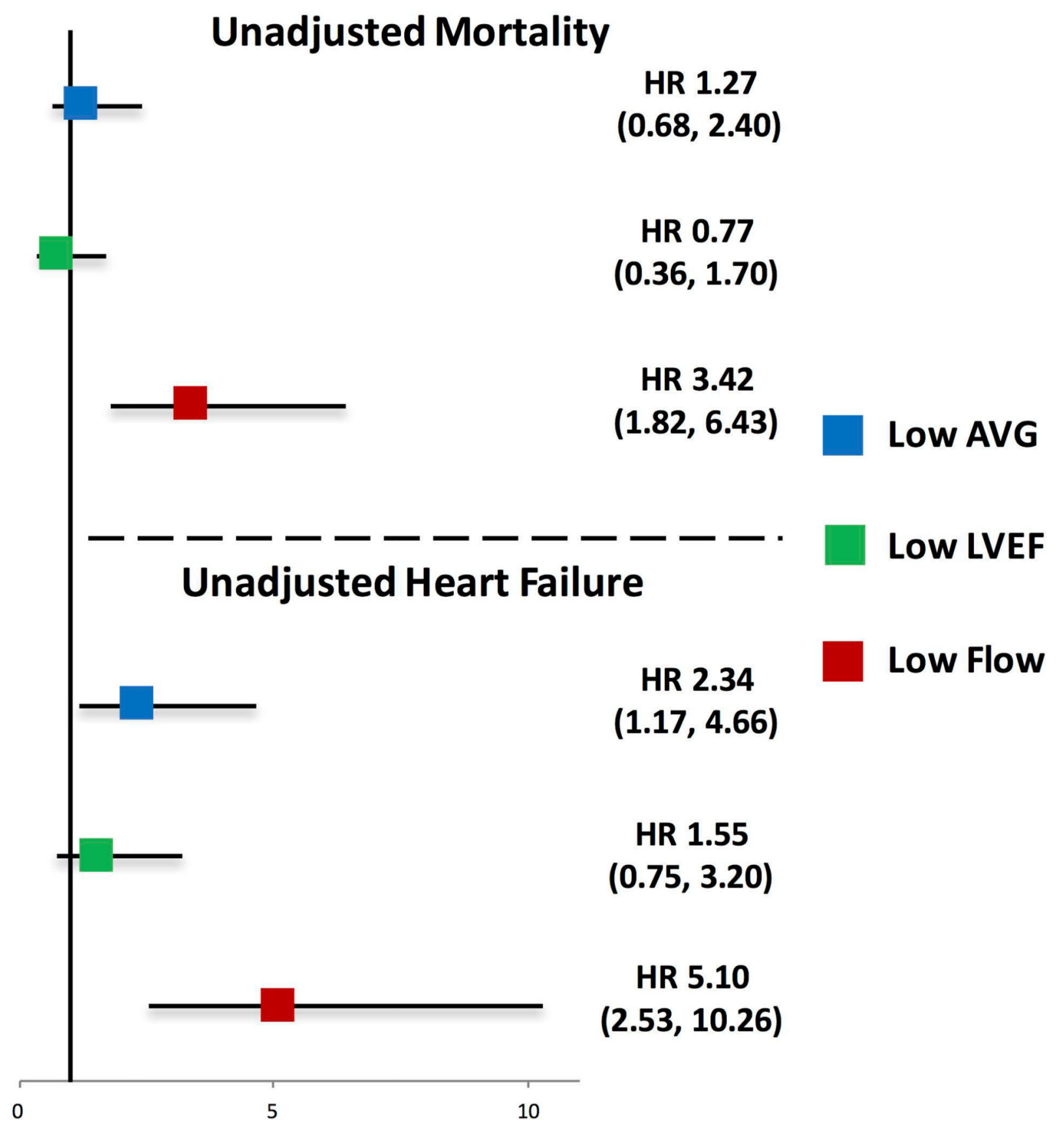

Figure 3. Unadjusted 1-year mortality and heart failure readmission Hazard ratios for low AVG, low LVEF, and low flor with respect to unadjusted mortality and heart failure readmission at 1 year after TAVR. HR, hazard ratio; AVG, aortic valve gradient; LVEF, left ventricular ejection fraction. 


\section{Unadjusted Mortality}

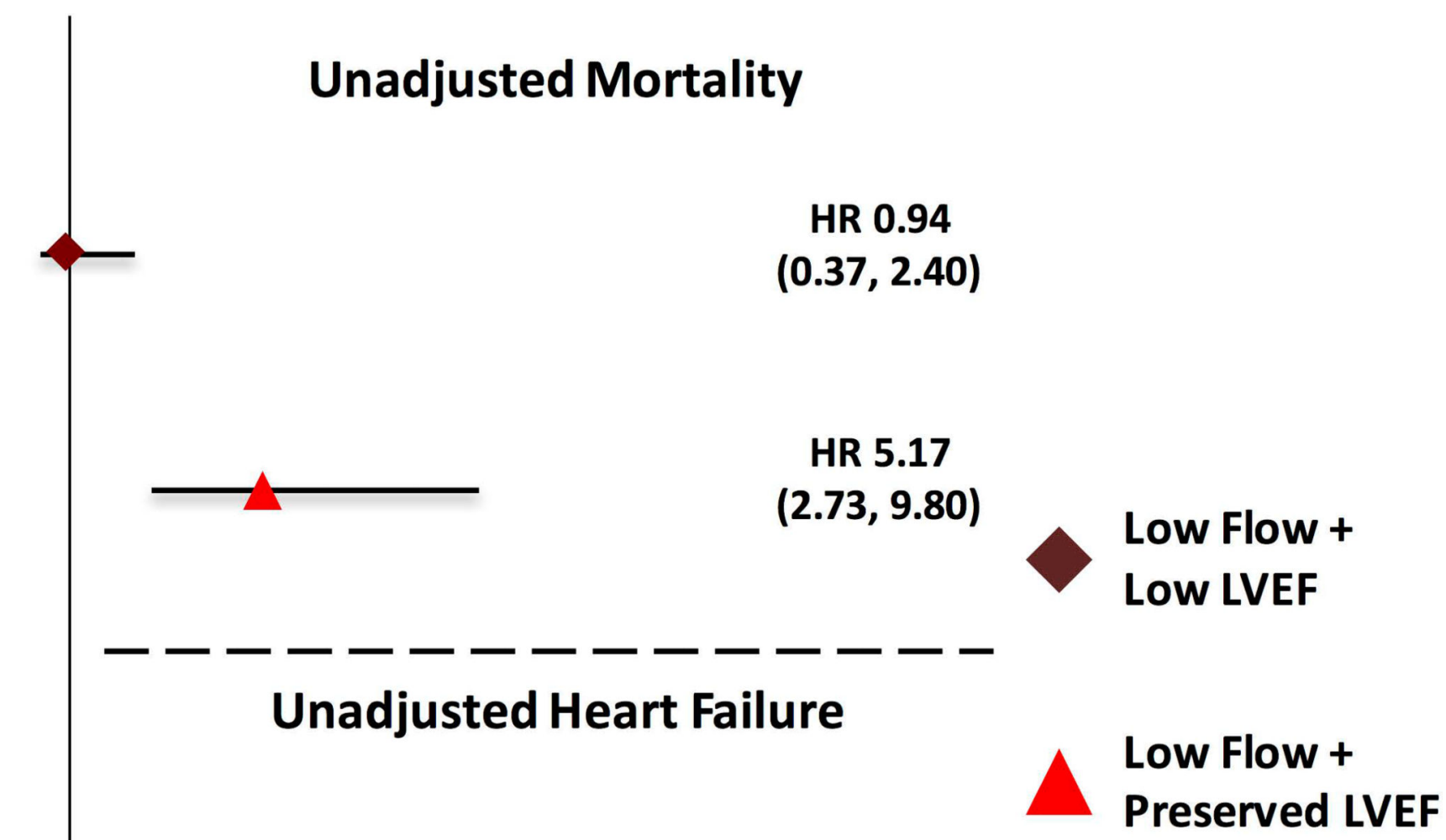

HR 1.15

$(0.44,2.98)$

HR 7.69

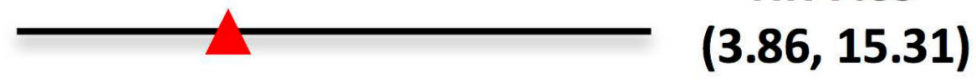

$(3.86,15.31)$

Figure 4. Unadjusted 1-year mortality and heart failure readmission

Hazard ratios low flow and preserved and low LVEF with respect to unadjusted mortality and heart failure readmission at 1 year after TAVR. HR, hazard ratio; LVEF, left ventricular ejection fraction. 


\section{Adjusted Mortality}

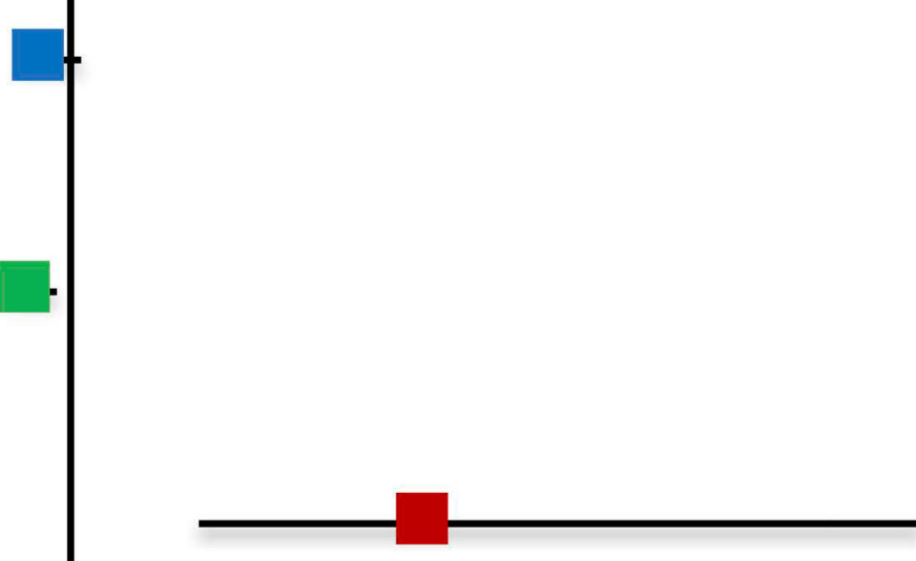

HR 0.56

$(0.26,1.20)$

HR 0.35

$(0.15,0.79)$

HR 6.51

$(2.98,14.22)$

HR 1.16

$(0.53,1.51)$

\section{- - - - - - - - - - - $\quad$ Low LVEF Adjusted Heart Failure}

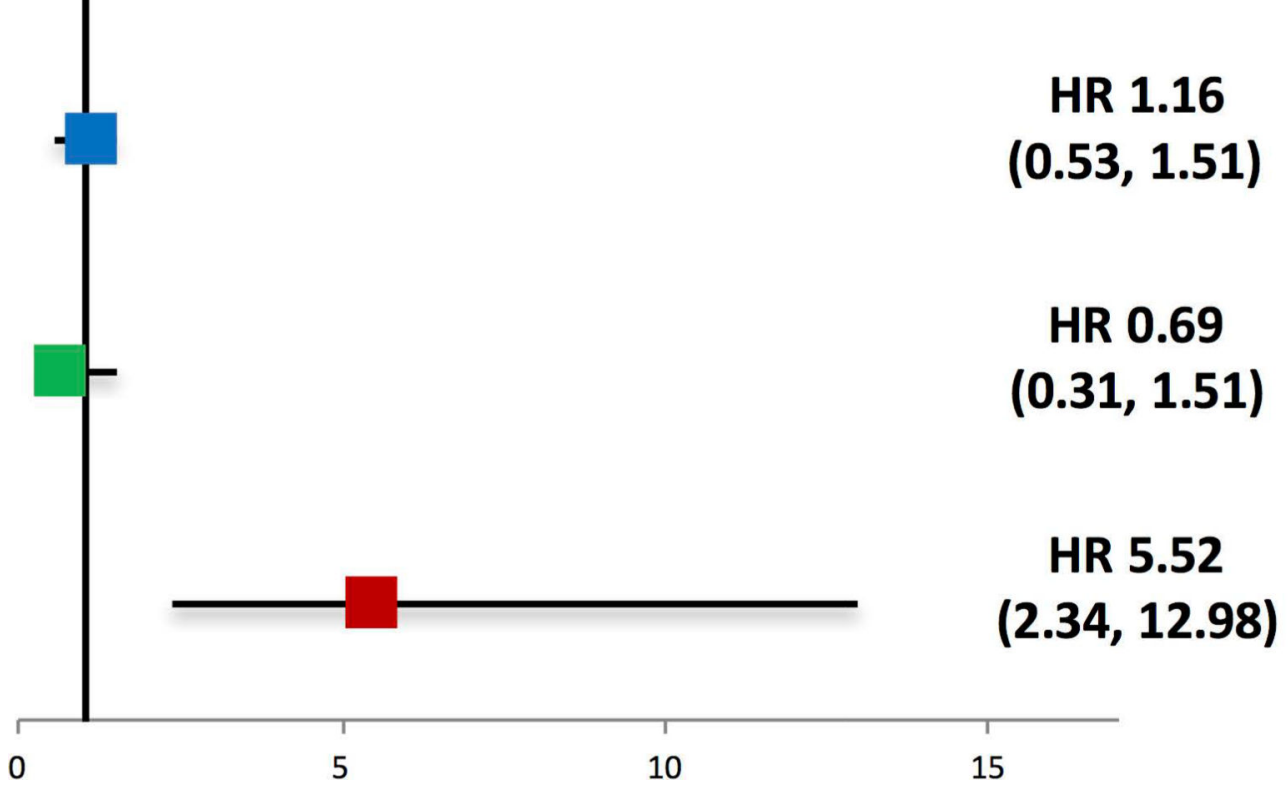

Figure 5. Adjusted 1-year mortality and heart failure readmission

Hazard ratios for low AVG, low LVEF, and low flor with respect to adjusted mortality and heart failure readmission at 1 year after TAVR. HR, hazard ratio; AVG, aortic valve gradient; LVEF, left ventricular ejection fraction. 
Table 1

Baseline Characteristics.

\begin{tabular}{|c|c|c|c|}
\hline & SVI $<35(n=96)$ & SVI $=>35(n=244)$ & $P$ value \\
\hline Age, $y$ & $81.6(9.3)$ & $81.6(8.3)$ & 0.97 \\
\hline Female sex & $44(45.8)$ & $128(27.1)$ & 0.27 \\
\hline Prior CABG & $30(31.3)$ & $73(29.9)$ & 0.81 \\
\hline Prior PCI & $33(34.4)$ & $64(26.2)$ & 0.13 \\
\hline Prior aortic valvular surgery & $17(17.7)$ & $41(16.8)$ & 0.84 \\
\hline Prior other valvular surgery & $6(6.3)$ & $6(2.5)$ & 0.088 \\
\hline CAD, \# diseased vessels & & & 0.75 \\
\hline $\mathbf{0}$ & $42(43.8)$ & $99(40.6)$ & \\
\hline 1 & $18(18.8)$ & $50(20.5)$ & \\
\hline 2 & $15(15.6)$ & $31(12.7)$ & \\
\hline 3 & $21(21.9)$ & $64(26.2)$ & \\
\hline Left main disease & $9(9.4)$ & $21(8.6)$ & 0.79 \\
\hline Peripheral arterial disease & $33(34.4)$ & $69(28.3)$ & 0.27 \\
\hline Hypertension & $85(88.5)$ & $228(93.4)$ & 0.13 \\
\hline Diabetes Mellitus & $40(41.7)$ & $97(39.8)$ & 0.75 \\
\hline Prior Pacemaker & $25(26.0)$ & $37(15.2)$ & 0.0194 \\
\hline Atrial fibrillation/ flutter & $48(50.0)$ & $107(43.9)$ & 0.31 \\
\hline Chronic lung disease & & & 0.61 \\
\hline Mild & $18(18.8)$ & $33(13.5)$ & \\
\hline Moderate & $14(14.6)$ & $32(13.1)$ & \\
\hline Severe & $12(12.5)$ & $32(13.1)$ & \\
\hline Prior stroke & $11(11.5)$ & $34(13.9)$ & 0.54 \\
\hline Prior stroke or TIA & $12(12.5)$ & $52(21.3)$ & 0.061 \\
\hline Baseline creatinine, $\mathrm{mg} / \mathrm{dL}$ & $1.50(0.98)$ & $1.32(0.96)$ & 0.12 \\
\hline On hemodialysis & $4(4.2)$ & $9(3.7)$ & 0.84 \\
\hline NYHA III or IV & $86(89.6)$ & $210(86.1)$ & 0.38 \\
\hline KCCQ-12 Score & $28.8(21.6)$ & $33.9(21.6)$ & 0.11 \\
\hline STS score & $8.4(6.1)$ & $7.3(3.5)$ & 0.077 \\
\hline AVA & $0.64(0.18)$ & $0.68(0.17)$ & 0.072 \\
\hline Peak gradient & $58.8(16.3)$ & $77.0(20.8)$ & $<0.0001$ \\
\hline Mean gradient & $35.1(10.9)$ & $46.0(11.7)$ & $<0.0001$ \\
\hline AVG $<40 \mathrm{mmHg}$ & $69(71.9)$ & $64(26.2)$ & $<0.0001$ \\
\hline LVEF & $45.9(15.5)$ & $55.4(12.0)$ & $<0.0001$ \\
\hline LVEF $<50 \%$ & $48(50.0)$ & $40(16.4)$ & $<0.0001$ \\
\hline AI, severe & $0(0.0)$ & $2(0.8)$ & 0.51 \\
\hline MR, severe & $5(5.2)$ & $15(6.2)$ & 0.20 \\
\hline TR, severe & $0(0.0)$ & $2(0.8)$ & 0.51 \\
\hline
\end{tabular}

Catheter Cardiovasc Interv. Author manuscript; available in PMC 2019 March 01. 


\begin{tabular}{|l|c|c|c|}
\hline & SVI<35 (n=96) & SVI=>35 (n=244) & P value \\
\hline Bypass used & $5(5.2)$ & $6(2.5)$ & 0.0197 \\
\hline Valve in valve & $13(13.5)$ & $38(15.6)$ & 0.64 \\
\hline Valve type & & & $<0.0001$ \\
\hline Sapien & $54(56.3)$ & $97(39.8)$ & \\
\hline Sapien XT & $20(20.8)$ & $76(31.2)$ & \\
\hline CoreValve & $18(18.8)$ & $33(13.5)$ & \\
\hline Sapien S3 & $4(4.2)$ & $38(15.6)$ & \\
\hline Access site & & & 0.46 \\
\hline Transfemoral & $72(75.0)$ & $186(76.2)$ & 0.52 \\
\hline Transaortic & $14(14.6)$ & $36(14.8)$ & \\
\hline Transapical & $7(7.3)$ & $17(7.0)$ & \\
\hline Other & $3(3.1)$ & $5(2.0)$ & \\
\hline
\end{tabular}

Categorical data are presented as number (percentage) and continuous data are presented as mean (standard deviation).

$\mathrm{Y}$, years; mg, milligrams; dL, deciliter; $\mathrm{CABG}$, coronary artery bypass grafting; $\mathrm{PCI}$, percutaneous coronary intervention; CAD, coronary artery disease; TIA, transient ischemic attack; NYHA, New York Heart Association; KCCQ, Kansas City Cardiomyopathy Questionnaire; STS, Society of Thoracic Surgeons; AVA, aortic valve area; AVG, aortic valve gradient; $\mathrm{mmHg}$, millimeters of mercury; LVEF, left ventricular ejection fraction; AI, aortic insufficiency; MR, mitral regurgitation; TR, tricuspid regurgitation. 


\section{Table 2}

\begin{tabular}{|c|c|c|c|}
\hline & Low Flow (n=96) & $\begin{array}{l}\text { Preserved Flow } \\
(n=244)\end{array}$ & P value \\
\hline Mortality & $12(12.5)$ & $5(2.1)$ & 0.0002 \\
\hline Stroke & $6(6.3)$ & $9(3.7)$ & 0.093 \\
\hline Change in creatinine & $-0.1(0.7)$ & $-0.07(0.4)$ & 0.67 \\
\hline Need for transfusion & $31(32.3)$ & $45(18.4)$ & 0.0029 \\
\hline Length of stay, days & $7.1(6.9)$ & $4.9(4.7)$ & 0.0041 \\
\hline ICU Hours & $63.5(106.9)$ & $35.7(84.2)$ & 0.024 \\
\hline
\end{tabular}

Categorical data are presented as number (percentage) and continuous data are presented as mean (standard deviation). 


\section{Table 3}

30-day outcomes.

\begin{tabular}{|l|l|l|l|}
\hline & Low Flow (n=96) & $\begin{array}{l}\text { Preserved Flow } \\
(\mathbf{n = 2 4 4})\end{array}$ & P value \\
\hline Death & $15(15.6)$ & $6(2.5)$ & $<0.0001$ \\
\hline HF Admission & $7(8.6)$ & $5(2.1)$ & 0.0113 \\
\hline
\end{tabular}

Values are presented as number (percentage). 